\title{
Imaging of Lipids, Metabolites and Drugs with Nanospray Desorption Electrospray Ionization Mass Spectrometry
}

Ingela Lanekoff ${ }^{1}$, Rosalie $\mathrm{Chu}^{2}$, Chistopher Anderton ${ }^{2}$, Christine Mahoney ${ }^{2}$, Ljiljana Paša-Tolic ${ }^{2}$, Jordan Smith $^{3}$, Charles Timchalk ${ }^{3}$, Mathew Thomas ${ }^{3}$, James Carson ${ }^{3}$, Kristin Burnum ${ }^{3}$, Allan Konopka ${ }^{3}$, and Julia Laskin ${ }^{1}$

1. Physical Sciences Division, PNNL, Richland, WA, USA.

2. EMSL, PNNL, Richland, WA, USA.

${ }^{3 .}$ Biological Sciences Division, PNNL, Richland, WA, USA.

Mass spectrometry imaging (MSI) enables simultaneous spatially-resolved analysis of numerous ionizable molecules on a sample surface. Ambient ionization techniques are attractive because they enable imaging without sample pretreatment. Nanospray desorption electrospray ionization, nano-DESI, is a novel ambient MSI technique developed at PNNL.[1] Nano-DESI utilizes localized desorption of analyte molecules from surfaces into a liquid bridge between two fused silica capillaries, the nano-DESI probe (Figure 1). In nano-DESI, a solvent supplied through the primary capillary to the surface is removed by the secondary capillary. Analyte molecules desorbed into the solvent are converted into ions via nanoelectrospray of the resulting solution and detected by a mass spectrometer. Ion images of molecules on the surface are created by continuously moving the sample under the nano-DESI probe and collecting mass spectra.[2] (Figure 1) In this presentation we will compare nano-DESI imaging with more traditional approaches including matrix assisted laser desorption ionization (MALDI) and secondary ion mass spectrometry (SIMS). Since the three imaging techniques rely on different ionization mechanisms and sample pretreatment requirements, we show that the combination of these techniques provides complementary characterization of the spatial distribution of lipids and metabolites in tissue samples.

We will present several examples of nano-DESI imaging of lipids and metabolites in biological samples. Specifically, we will introduce an approach for efficient detection and online quantification of drugs and metabolites in brain and other tissue samples and demonstrate spatial profiling of metabolites and lipids in living microbial communities.

Nicotine is an addictive drug affecting the brain's reward system by activating the cholinergic system. The localization of nicotine is especially important since its effect on brain chemistry may contribute to adverse neurological effects and increased knowledge will aid in computational modeling to predict the implications of regional brain dosimetry. To accurately measure the spatial distribution of nicotine in the brain of an exposed rat we imaged a section of the rat brain using a nano-DESI solvent doped with deuterated nicotine as an internal standard. Because both nicotine and deuterated nicotine experience similar ion suppression effects from endogenous substances in the rat brain, addition of the deuterated standard to the nano-DESI solvent enabled accurate determination of the nicotine distribution in rat brain tissue sections and facilitated relative quantification of nicotine in the rat brain.[3] Using this approach, we demonstrated subfemtomole sensitivity of nano-DESI imaging.

In nature, phototrophic biofilms of cyanobacteria are known to live in complex microbial mats in symbiosis with other bacteria. To further understand this companionship it is important to know what compounds cyanobacteria secrete and share. Synechococcus sp. PCC 7002 is a marine bacterium 
cultivated on $\mathrm{A}+$ agar with high salt content $(\sim 300 \mathrm{mM})$. Since nano-DESI does not require any sample preparation, chemical gradients of metabolites being produced by Synechococcus sp. PCC 7002 colonies were profiled on living colonies cultivated on A+ agar. The results show the evolution of metabolite gradients of glucosylglycerol and sucrose, generated on the agar over a time course of 6 days and demonstrate the unique power of nano-DESI for chemical characterization of living microbial systems.[4] [5] (Figure 2)
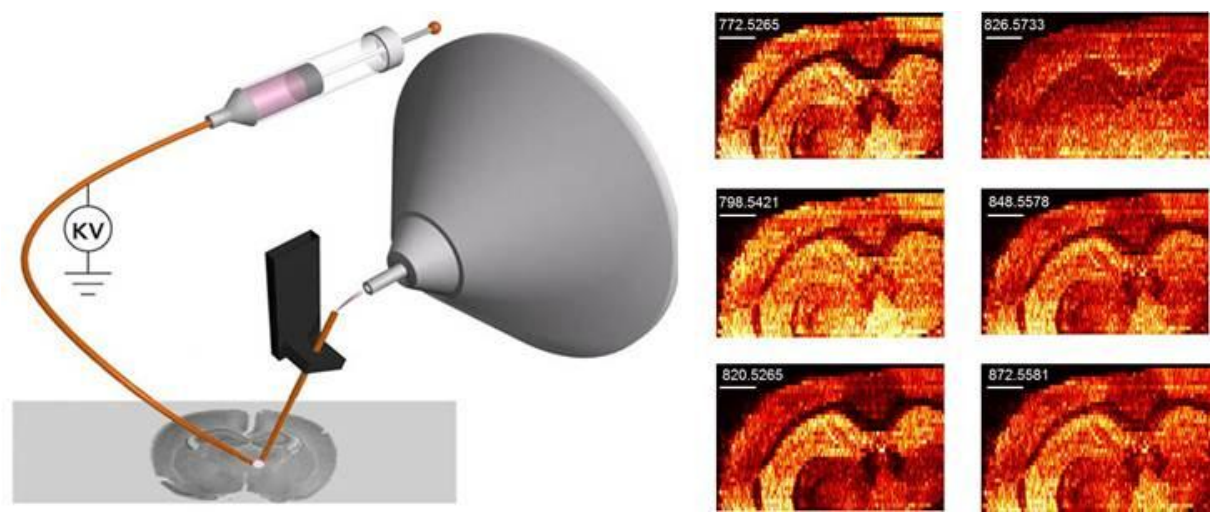

Figure 1. The nano-DESI setup and images of some phosphatidylcholine species, PC $32: 0(\mathrm{~m} / \mathrm{z}$ 772.5265), PC 34:1 ( $\mathrm{m} / z$ 798.5421), PC 36:4 ( $\mathrm{m} / \mathrm{z} 820.5265)$, PC 36:1 ( $\mathrm{m} / \mathrm{z} 826.5733)$, PC $38: 4(\mathrm{~m} / z$ 848.5578), PC 40:6 ( $\mathrm{m} / \mathrm{z} 872.5581)$. Scale bar shows $2 \mathrm{~mm}$.

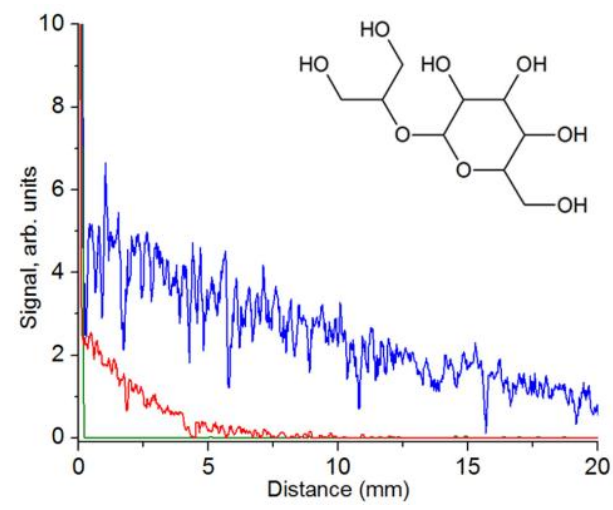

Figure 2. Diffusion gradient of glucosylglycerol from Synechococcus sp. PCC 7002 on A+ agar. Traces are color coded with age of colony. Blue $-144 \mathrm{~h}$, red -72 hours and green -48 hours.

[1] PJ Roach, J Laskin, A Laskin et al. Analyst 135 (2010), p. 2233

[2] I Lanekoff, BS Heath, A Liyu, M Thomas, JP Carson, J Laskin. Anal. Chem. 84 (2012), p. 8351

[3] I Lanekoff, M Thomas, JP Carson, JN Smith, C Timchalk, J Laskin. Anal. Chem. 85 (2013), p. 882

[4] I Lanekoff, O Geydebrekhy, GE Pinchuk, AE Konopka, J Laskin. Analyst DOI: 10.1039/C3AN36716A

[5] - The research described in this presentation is part of the Chemical Imaging Initiative, at Pacific Northwest National Laboratory (PNNL). It was conducted under the Laboratory Directed Research and Development Program at PNNL a multiprogram national laboratory operated by Battelle for the U.S. Department of Energy (DOE) under Contract DE-AC05-76RL01830. The research was performed at EMSL, a national scientific user facility sponsored by the DOE's Office of Biological and Environmental Research and located at PNNL. 